\title{
Splicing Hollow-Core Photonic Bandgap Fibers to Step- Index Fibers Using an Arc Fusion Splicer
}

\author{
R. Thapa, K. L. Corwin, B. R. Washburn \\ Kansas State University, Department of Physics, 116 Cardwell Hall, Manhattan, KS 66506 \\ emailwashburn@phys.ksu.edu, phone: 785-532-2263,fax: 785-532-6606
}

\begin{abstract}
A method for splicing hollow-core photonic bandgap fiber to step-index fiber using a commercial arc fusion splicer has been developed. These splices compare favorably with commercially available splices for nonlinear optical interactions with gases.

(C) 2005 Optical Society of America

OCIS codes: (230.3990) Microstructure devices; (060.2310) Fiber optics; (060.4510) Optical communications; (120.3930)

Metrological Instrumentation; (300.1030) Absorption;
\end{abstract}

\section{Introduction}

The advent of photonic bandgap fiber (PBG) has spurred many studies of nonlinear interactions between light and molecular gases. For instance, Raman scattering by hydrogen gas in these fibers allows highly efficient wavelength conversion [1]. Electromagnetically-induced transparency has been observed in acetylene-filled fibers with applications toward all-optical fiber communications [2]. Saturation spectroscopy, for higher-accuracy portable optical frequency references, has also been demonstrated [3]. Even linear interactions are significantly enhanced, resulting in the development of gas sensors [4] and Doppler- and pressure-broadened frequency references [1].

Practical devices based on gas-filled PBG fiber require both ends of the hollow fiber to be sealed. Typically this is accomplished by fusion splicing to solid-core single mode fiber (SMF), which is challenging due to the fragile PBG fiber. In fact, PBG fibers have been sealed after being filled by acetylene gas [1], but this technique relies on the use of an expensive, filament-based fusion splicer. Commercially-available spliced PBG fibers are made with the same technology [5]. Solid-core microstructured fibers have been spliced with arc-splicers [6], and the collapse of air holes in PBG fiber for selective injection has been systematically investigated [7]. However, a simple recipe for splicing PBG fiber to SMF using a conventional arc fusion splicer has until now been lacking. Here we demonstrate a repeatable, robust, low loss $(<1.4 \mathrm{~dB})$ splice between a hollow-core PBG fibers and SMF. The performance of this fiber compares favorably with a commercially made spliced fiber for saturation spectroscopy.

\section{Fusion splicing hollow-core PBG fiber to SMF}

The reason why filament splicers are generally preferred for PBG fiber fusion splicing is that they heat more slowly and uniformly. Thus we wish to mimic the filament fusion splicer using an Ericsson FSU-995 electric arc fusion splicer. A multi-step splice procedure was used that involved 1) a short, high current arc and 2) a long, low current arc. Figure 1(a) shows the splicing setup and (b) shows the resulting PBG-SMF splice between a hollow-core PBG of core size $10 \mu \mathrm{m}$ and Coring SMF-28e ${ }^{\circledR}$.
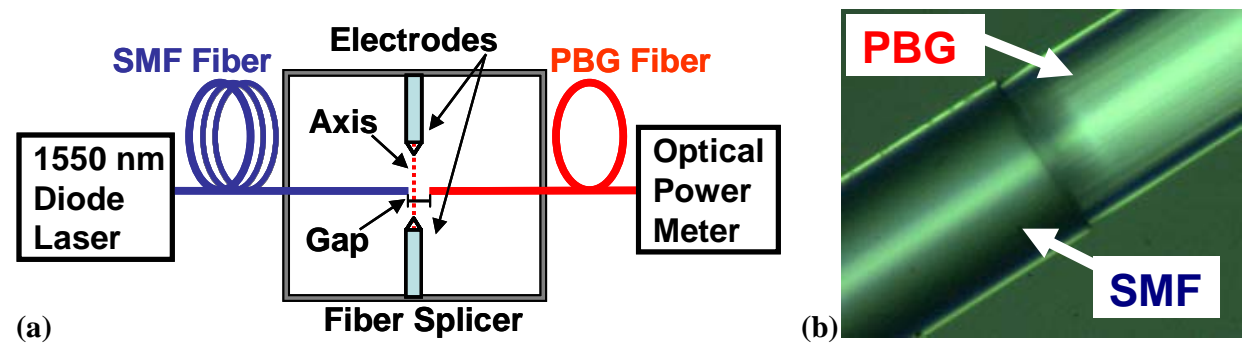

Fig. 1. (a) Experimental set-up for the measurement of splice losses. A tunable diode laser at $1550 \mathrm{~nm}$ is used with an optical power meter to measure the coupling during the splice procedure using a commercial arc splicer. (b) A micrograph showing the splice between the SMF and PBG fiber. Picture courtesy of the GaN Group at KSU Physics.

The loss with respect to the butt-coupled throughput was measured during the splicing procedure (Fig. 2). During the splice program the two fibers were briefly butt-coupled (touch point) and then a gap of $+10 \mu \mathrm{m}$ was made. The center position was set to 260, thus the offset of the gap center from the electrodes axis was roughly $5 \mu \mathrm{m}$, according to the calibration from Ref. [7]. This offset ensured that less heat was applied to the PBG fiber and prevented a collapse of the air holes. The first fusion current of $10 \mathrm{~mA}$ was used for $0.2 \mathrm{~s}$, and then a lower $7 \mathrm{~mA}$ current was used for $12 \mathrm{~s}$. During the second current the fibers were overlapped with a gap width (overlap) of 
-15 $\mu \mathrm{m}$. (Negative gaps indicate that the fibers are pushed further together than they were when butt-coupled.) Afterwards an independent measurement determined a total loss of $1.4 \mathrm{~dB}$. The splice was mechanically strong and could be bent in a $\sim 1.5 \mathrm{~cm}$ circular radius before breaking.

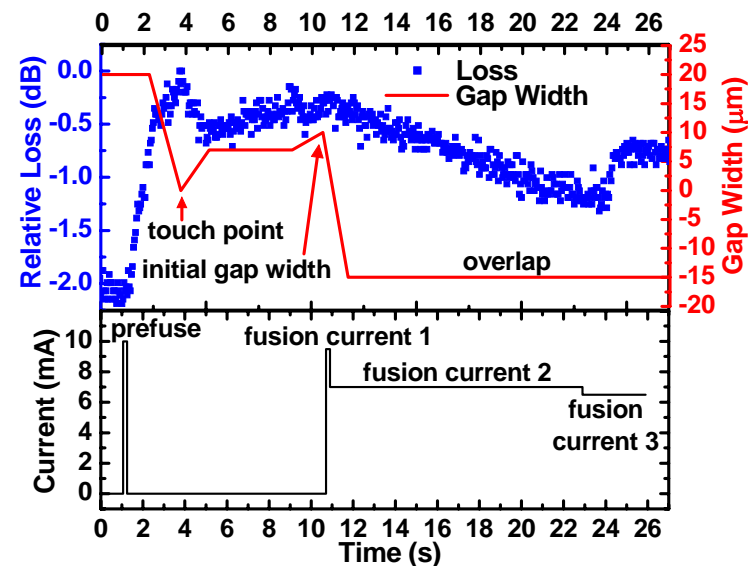

\begin{tabular}{|ll|}
\hline $\begin{array}{ll}\text { Splice Parameters: } \\
\text { Initial Gap Width }\end{array}$ & $10 \mu \mathrm{m}$ \\
Overlap & $-15 \mu \mathrm{m}$ \\
Fusion time 1 & $0.2 \mathrm{~s}$ \\
Fusion current 1 & $10 \mathrm{~mA}$ \\
Fusion time 2 & $12 \mathrm{~s}$ \\
Fusion current 2 & $7 \mathrm{~mA}$ \\
Fusion time 3 & $3 \mathrm{~s}$ \\
Fusion Current & $6.5 \mathrm{~mA}$ \\
Center position $\quad 260$ \\
(gap center was offset from \\
the electrodes axis by $\sim 5 \mathrm{~m}$ )
\end{tabular}

Fig. 2. The measured loss with respect to the butt-couple throughput between the SMF and PBG fiber during the fusion procedure. The gap width was estimated during the time from 10.5 to $11.7 \mathrm{~s}$.

\section{Hollow-core PBG fiber to SMF splice for a gas-filled optical frequency reference}

To demonstrate the utility of the splice, a $0.78 \mathrm{~m}$ length of PBG fiber spliced to SMF (called Fiber 1) was evacuated by pumping with a mechanical rough pump on the PBG fiber's open end in a vacuum chamber (Fig. 1(a)). The fiber was evacuated to $15 \mathrm{mT}$ over 12 hours. Then the PBG fiber was filled with acetylene gas in the same vacuum chamber to a pressure of $860 \mathrm{mT}$. Absorption spectroscopy was performed on the gas in the PBG fiber and a strong absorption feature was observed. The spectra were compared to the same measurement performed with a PBG fiber that had a splice made using a filament fusion splicer (called Fiber 2, purchased from Crystal Fibre). The $2 \mathrm{~m}$ length of PBG fiber in Fiber 2 was at 320 mT. Both gas-filled fibers exhibited similar absorption (Fig. 3(b)).

Unfortunately, interference with light reflected from the splice limited the visibility of the saturated absorption feature. The exact pathways interfering are not known, but the Fabry-Perot-like fringes had a contrast ratio of 6\% (Fiber 1) and 7\% (Fiber 2), indicating the similarity between the two splices.

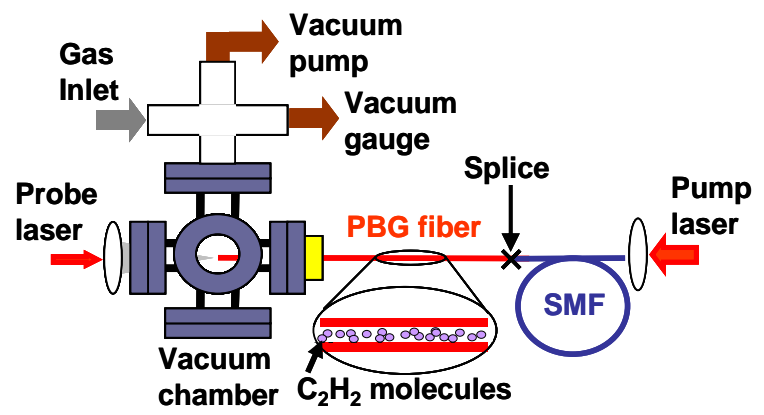

(a)

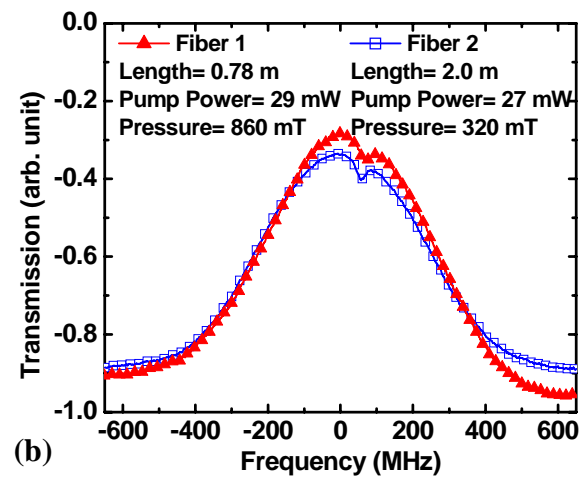

(b)

Fig. 3. Chamber used to evacuate and fill the PBG fiber with acetylene gas for the saturated absorption spectroscopy. (b) Saturated absorption spectra of acetylene filled PBG fibers. The fiber with the arc fusion splice (Fiber 1) exhibits a similar absorption profile as the fiber with the filament fusion splice (Fiber 2).

\section{References}

[1] F. Benabid, F. Couny, J. C. Knight, T. A. Birks, and P. S. J. Russel, "Compact, stable and efficient all-fibre gas cells using hollow-core photonic crystal fibres," Nature 434, 488-491 (2005).

[2] S. Ghosh, J. E. Sharping, D. G. Ouzounov, and A. L. Gaeta, "Resonant optical interactions with molecules confined in photonic band-gap fibers," Phys. Rev. Lett. 94, 093902-1, (2005).

[3] M. Faheem, R. Thapa, and K. L. Corwin, "Spectral hole burning of acetylene gas inside a photonic bandgap optical fiber," in Conference on Lasers and Electro-optics Technical Digest, (Optical Society of America, Washington, D.C., 2005), CWJ6.

[4] T. Ritari, J. Tuominen, H. Ludvigsen, J. C. Petersen, T. Sørensen, T. P. Hansen, and J. C. Simonsen, "Gas sensing using air-guiding photonic bandgap fibers," Opt. Express 12, 4081-4087 (2004).

[5] Crystal Fibre A/S, http://www.crystal-fibre.com/support/faq.shtm.

[6] B. Bourliaguet, C. Paré, F. Émond, A. Croteau, A. Prouix, and R. Vallée, "Microstructured fiber splicing," Opt. Express 11, $3412-17$ (2003).

[7] L. Xiao, W. Jin, M. S. Demokan, H. L. Ho, Y. L. Hoo, and C. Zhao, "Fabrication of selective injection microstructured optical fibers with a conventional fusion splicer," Opt. Express 13, 9014-9022 (2005). 